1682 


\title{
Romantik \\ in Deutschland
}

Ein interdisziplinäres

Symposion

Herausgegeben

von Richard Brinkmann

\author{
Sonderband der \\ "Deutschen Vierteljahrsschrift \\ für Literaturwissenschaft \\ und \\ Geistesgeschichte" \\ Mit 49 Abbildungen \\ J.B. Metzlersche \\ Verlagsbuchhandlung \\ Stuttgart
}




\section{CIP-Kurztitelaufnahme der Deutschen Bibliothek}

Romantik in Deutschland: e. interdisziplinäres Symposion / hrsg. von Richard Brinkmann.

- 1. Aufl. - Stuttgart: Metzler, 1978.

(Deutsche Vierteljahrsschrift für Literaturwissenschaft und Geistesgeschichte; Sonderbd.)

ISBN 978-3-476-00404-8

NE: Brinkmann, Richard [Hrsg.]

ISBN 978-3-476-00404-8

ISBN 978-3-476-04397-9 (eBook)

DOI $10.1007 / 978-3-476-04397-9$

(C) Springer-Verlag GmbH Deutschland 1978

Ursprünglich erschienen bei J.B. Metzlersche Verlagsbuchhandlung und Carl Ernst Poeschel Verlag GmbH in Stuttgart 1978 


\section{INHALT}

Vorbemerkungen des Herausgebers

VII

Zur Eröffnung des Symposions . . . . . . . . . . . . . . . . . . .

RICHARD BRINKMANN (Tübingen): Romantik als Herausforderung. Zu ihrer wissenschaftsgeschichtlichen Rezeption. Einführungsvortrag . . . . . . . . .

Romantik - im Spannungsfeld von sozialem Wandel und Stagnation

Wolfgang Schieder (Trier): Einführendes Referat

GÜNTER BIRTSCH (Trier): Aspekte und Wandlungen des Freiheitsbegriffs in der deutschen Romantik zwischen naturrechtlichem Rationalismus und Traditionalismus . . . . . . . . . . . . . . . . . . . . . . .

KarL-Georg Faber (Münster): Zur Machttheorie der politischen Romantik und der Restauration . . . . . . . . . . . . . . . . . . . . .

ULRICH SCHEUNER (Bonn): Staatsbild und politische Form in der romantischen Anschauung in Deutschland ..............

VOLKer StanSLOWSK (Hamburg): Bürgerliche Gesellschaft als Organismus. Zum Verhältnis von Staats- und Naturwissenschaften in der "Politischen Romantik"

Friedrich A. Kittler (Freiburg i. Br.): Der Dichter, die Mutter, das Kind. Zur romantischen Erfindung der Sexualität . . . . . . . . . . . . . .

Otto DanN (Köln): Gruppenbildung und gesellschaftliche Organisierung in der Epoche der deutschen Romantik . . . . . . . . . . . . . . . . . . .

ERnSt. Hanisch (Salzburg): Der "vormoderne" Antikapitalismus der Politischen Romantik. Das Beispiel Adam Müller . . . . . . . . . . . . . . . . . .

Ulrich Stadler (Basel): Die Auffassung vom Gelde bei Friedrich von Hardenberg (Novalis) . . . . . . . . . . . . . . . . . . .

Christof Dipper (Trier): Diskussionsbericht

Romantik - im Spannungsfeld von Naturgefühl, Naturwissenschaft und Naturphilosophie

Dietrich v. Engelhardt (Heidelberg): Einführendes Referat

John Neubauer (Pittsburgh): Zwischen Natur und mathematischer Abstraktion: der Potenzbegriff in der Frühromantik . . . . . . . . . . . . . . . . . .

Henricus Adrianus Marie Snelders (Utrecht): Atomismus und Dynamismus im Zeitalter der Deutschen Romantischen Naturphilosophie . . . . . . . .

THEODORE FELDMAN (Berkeley): Josef Weber : A Transitional Figure of the Bavarian Enlightenment and Romantic . . . . . . . . . . . . . . . . . .

Hans Querner (Heidelberg): Ordnungsprinzipien und Ordnungsmethoden in der Naturgeschichte der Romantik (mit 2 Abb.) . . . . . . . . . . . . . . . .

HeINRICH SCHIPPERges (Heidelberg): Krankwerden und Gesundsein bei Novalis

KarL Ed. Rothschun (Münster): Naturphilosophische Konzepte der Medizin aus der Zeit der deutschen Romantik (mit 3 Abb.) . . . . . . . . . . . . . . .

WULF SEgebrecht (Regensburg): Krankheit und Gesellschaft. Zu E. T. A. Hoffmanns Rezeption der Bamberger Medizin . . . . . . . . . . . . . . .

Dorothea Kunn (Marbach a. N.): Diskussionsbericht . . . . . . . . . . .

DiETRICH VON ENGELHARDT (Heidelberg): Bibliographie der Sekundärliteratur zur romantischen Naturforschung und Medizin 1950-1975

Romantik - im Spannungsfeld von Kunstglaube, Mythologie und Theologie I

Hans-JoAChim Mähl (Kiel): Einführendes Referat . . . . . . . . . . . Otto Poggeler (Bochum): Die neue Mythologie. Grenzen der Brauchbarkeit des deutschen Romantik-Begriffs . . . . . . . . . . . . . . 
HANNELORE LiNK $(\dagger)$ (München): Zur Fichte-Rezeption in der Frühromantik . . Friedrich Strack (Heidelberg): Die "göttliche" Kunst und ihre Sprache. Zum Kunst- und Religionsbegriff bei Wackenroder, Tieck und Novalis

Heinz Paetzold (Hamburg): Kunst als Organon der Philosophie. Zur Problematik des ästhetischen Absolutismus

WALTER Ch. ZIMMERLI (Zürich): Schellings "Deduktion eines allgemeinen Organons der Philosophie" als Bindeglied zwischen romantischer Kunstauffassung und der Neubegründung der Dialektik in Hegels Jenaer Philosophie . . . . . . .

SibyLle v. SteInsDorfF (München): Diskussionsbericht

Romantik - im Spannungsfeld von Kunstglaube, Mythologie und Theologie II

Wolfgang Frunwald (München): Einführendes Referat . . . . . . . . . .

Hermann Timm (Heidelberg): Universalität und Individuation. Das Konzept des frühromantischen "Christianismus" . . . . . . . . . . . . . . . . . . .

Elisabeth Stopp (Cambridge): Romantic Affinities of Johann Michael Sailer's Kerygmatic Writing .

PhILIPP SChäFER (Rottenburg a. N.): Der Weg der Auseinandersetzung mit dem Rationalismus der Aufklärung zum Verständnis der Kirche als lebendige Gemeinschaft. Ein Beitrag der katholischen Theologie zur Romantik. . . . .

BernhardCAspfr (Augsburg): Der historische Besen. Oder über die Geschichtsauffassung in E. T. A. Hoffmanns Serapionsbrüdern und in der Katholischen Tübinger Schule . . . . . . . . . . . . . . . . . . . .

ERnst Lichtenhahn (Basel): Zur Idee des goldenen Zeitalters in der Musikanschauung E. T. A. Hoffmanns . . . . . . . . . . . . . . . . . . . . . . 502

Sibylle v. Steinsdorff (München): Diskussionsbericht 513

Romantik - im Spannungsfeld von Kunst, Ästhetik und Wirklichkeit

Walter MÚller-Seidel (München): Einführendes Referat . . . . . . . . .

Gonthier-Louis FinK (Straßburg): Volk und Volksdichtung in der ersten Berliner Romantik

MANFREd Frank (Düsseldorf): Schleiermachers hermeneutische Sprachtheorie und das Problem der Divination . . . . . . . . . . . . . . . . . . . . .

VıoRICA NişCov (Bukarest): Das Fragment als Absicht und Durchführung, als Plurivalenz und Eindeutigkeit. Glauben und Liebe von Novalis

ERnSt Behler (Seattle): Die Theorie der Tragödie in der deutschen Frühromantik

Peter v. Matt (Zürich): Gespaltene Liebe. Die Polarisierung von erotischer und geistlicher Lyrik als Strukturprinzip des romantischen Gedichts . . . . . . .

HaRTwig Schultz (Frankfurt a. M.): Zur Form der romantischen Lyrik. Beobachtungen am Vers Eichendorffs.

Gerhard Schulz (Melbourne): Die metaphorische Darstellung des Gegensatzes Einsamkeit - Offentlichkeit in der deutschen romantischen Lyrik . . . . . . .

Klaus Kanzog (München): Formel, Motiv, Requisit und Zeichen bei E. T. A. Hoffmann . . . . . . . . . . . . . . . . . . . . . . . .

Wolfhart Henckmann (München): Symbol und Allegorie bei K. W. F. Solger Peter-Klaus Schuster (Hamburg) und Konrad Feilchenfeldt (München): Philipp Otto Runges Vier Zeiten und die Temperamentenlehre (mit 16 Abb.) . . 652

KonRad Feilchenfeldt (München): Diskussionsbericht . . . . . . . . . . 670

Klaus Lankheit (Karlsruhe): Caspar David Friedrich. Vortrag (mit 28 Abb.) 683 


\section{Vorbemerkungen des Herausgebers}

Vom 6.-9. September 1977 fand im Internationalen Institut für wissenschaftliche Zusammenarbeit auf Schloß Reisensburg bei Günzburg ein internationales und interdisziplinäres Symposion zur Romantik in Deutschland statt, das im Auftrag der Deutschen Forschungsgemeinschaft von deren Germanistischer Kommission veranstaltet wurde. Es war das zweite - das erste von der Grundkonzeption her interdisziplinär ausgerichtete - in einer Reihe von wissenschaftlichen Kolloquien, die auf Anregung von Albrecht Schöne im Jahre 1974 mit einem Symposion zum Barock begann und zu anderen Themen fortgesetzt wird.

Der Typus dieser Veranstaltung hat sich bewährt: Durch Ausschreibung in einschlägigen Zeitschriften sowie durch Rundbriefe an Institute und fachlich zuständige Wissenschaftler im In- und Ausland wurde das Unternehmen bekannt gemacht. Zugleich mit der Anmeldung sollte von den Interessenten das Exposé eines geplanten Beitrags eingereicht werden. Die "Kuratoren" der einzelhen Abschnitte des Symposions, die durch Themengruppen bestimmt waren, bemühten sich zusammen mit dem Leiter, aus den Vorschlägen die im sachlichen Kontext des Ganzen geeignetsten auszuwählen. Der Teilnehmerzahl waren von der Funktionsfähigkeit einer Diskussionsrunde und der Finanzierbarkeit her - die DFG trug die Organisations-, Reise- und Aufenthaltskosten - Grenzen gesetzt. Mehr als vierzig Teilnehmer konnten außer den Veranstaltern nicht eingeladen werden: etwa die Hälfte derer, die Entwürfe eingesandt hatten. Die endgültigen Vorlagen wurden zwei Monate vor dem Kolloquium in Kopien an alle Teilnehmer verschickt. Außer einem Einleitungs- und einem Abendvortrag, knappen Einführungsreferaten der "Kuratoren" und jeweils Abstract-artigen Zusammenfassungen der Beiträge durch ihre Verfasser blieb die Tagung ausschließlich der Diskussion vorbehalten.

Die Veranstalter hatten weder die Absicht noch die Illusion, irgendeine Art von voraufentworfenem Plan oder System durch angebotene oder auch angeregte Arbeiten gewissermaßen auszufüllen. Gleichwohl hielten sie für sinnvoll und nötig, thematische Rahmen zu setzen und durch eine - ergänzungsbedürftige - Auswahl stichworthafter Hinweise Impulse zu geben. Es ist wohl nicht überflüssig, die Arbeitsgebiete und die ihnen in der Ausschreibung zugeordneten Kataloge von denkbaren Sujets hier vorzustellen:

1. Romantik - im Spannungsfeld von sozialem Wandel und Stagnation ("Kurator": Prof. Dr. Wolfgang Schieder, Trier; "Redaktor"; Dr. Christoph Dipper, Trier). Kritik der politischen Romantik: Die Französische Revolution als historische Grunderfahrung / Organische Staatslehre / Familie als sozialer Grundbegriff / Säkularisation und Kirchenkrise / Entdeckung des Mittelalters / Bürgerlicher Nationalismus / Historische Wurzeln des Konservativismus / Vormoderner Antikapitalismus / Heilige Allianz / System der Restauration / Formen des Historismus. - Romantik und soziale Welt: Adel und Adelskrise / Bürger und Gebildete / Juden und Judenemanzipation / Vierter Stand und soziale Frage / Frau und Familie. - Romantik und Institutionen in Staat und Gesellschaft: Zensur und 
Öffentlichkeit / Schule und Gymnasium / Universitätsreformen / Fürsorge und Krankenpflege / Bünde und Vereine / Kirchliche Orden / Heiligenverehrung und Wallfahrten / Wartburg-Fest / Kölner Dombaufest von 1842.

2. Romantik - im Spannungsfeld von Kunst, Ästhetik und Wirklichkeit ("Kurator": Prof. Dr. Walter Müller-Seidel, München; "Redaktor": Dr. Konrad Feilchenfeldt, München).

Ästhetische Wahrnehmung und innere Wirklichkeit: Schwermut, Wehmut, Melancholie / Zur sogenannten wechselseitigen Einwirkung der Künste / Künstlergestalten und ihre Psyche (Wackenroder, E. T. A. Hoffmann) / Ästhetik und Pathologie / Das Unbewußte als Gegenstand wissenschaftlicher Kooperation / Synästhesie: Der Sinn des Begriffs / Die Ästhetik der Abstraktion. - Poetologie und Sprache: Wissenschaftssprache, Fachsprache / Verslehre / Sprachtheorie / Bildlichkeit (Allegorie, Symbol, Arabeske, Hieroglyphe) / Fragment / Geschichtsdichtung ("Philosophie der Weltalter") / Naturlyrik / Einsamkeit und Öffentlichkeit / Dichtung und Volk / Drama und Theater / Romantisch, Roman, Romantiker: Der Sinn der Begriffe / "Poetik" des Briefs. - Tradition und Aktualisierung: Formen und Strukturen / Stoffe und Motive / Traditionsvermittlung (Übersetzung, Edition, Satire) / Autorenkollektive (Der Doppelroman der Berliner Romantik) / Zeitdichtung (Gelegenheits-, Zeitgedichte) / Tendenzliteratur (Pamphlet, Flugschrift, Zeitungsartikel).

3. Romantik - im Spannungsfeld von Kunstglaube, Mythologie und Theologie ("Kuratoren": Prof. Dr. Hans-Joachim Mähl, Kiel, Prof. Dr. Wolfgang Frühwald, München; "Redaktor": Dr. Sibylle von Steinsdorf, München).

Universalismus der Frühromantik / Wackenroders Kunstreligion / Die Jenaer "ästhetische Kirche"/ Religiöser Sinn und Kunstsinn / Wandlungen im Verständnis des Mittelalters / Wechselbeziehungen zwischen Philosophie und Literatur / Fichtes Wissenschaftslehre und die Französische Revolution / Novalis' Fichteanismus / Kunst als Organon der Philosophie / Der Einfluß des Pietismus auf die Romantik / Die Erweckungsbewegung / Der Sailer-Kreis / Möhlers Symbolik / Die Leben-Jesu-Forschung / Romantiker als Anreger des Berliner Neupietismus / "Künstliche" und "natürliche" Mythologie / Mythenforschung (Görres, Creuzer) / Philosophie der Mythologie / Rezeption und Kritik der romantischen Mythenforschung / Mythologie und Märchen / Schuberts Einfluß auf das Bild der "Nacht" / Esoterik und Öffentlichkeitsbezug im Sprach- und Bildbereich usf.

4. Romantik im Spannungsfeld von Naturgefühl, Naturwissenschaft und Naturphilosophie ("Kurator": Prof. Dr. Dietrich von Engelhardt, Heidelberg, "Redaktor": Dr. Dorothea Kuhn, Marbach).

Romantische Naturforschung und Medizin zwischen idealistischer Philosophie und empirischer Wissenschaft / Naturbegriff; Welt als Organismus; Naturreiche und ihre Verwandtschaften (Systematik, Entwicklungslehren, Analogievorstellungen) / Kräfte in der Natur (Attraktion und Repulsion, Lebenskraft, Bildungstrieb, Sensibilität, Irritabilität und Reproduktionskraft) / Atomismus und Dynamismus; Polarität und Steigerung / Galvanismus und tierischer Magnetismus / Pflanze und Tier (Stufenleiter und Genese) / Anthropologie / Gesundheit, Krankheit 
und Tod / Kreislauf des Blutes; System der Krankheiten; Fieberlehre; Therapie / Nachtseite der Natur (Un- und Unterbewußtes, Traum, Tod, Somnambulismus, Geisterseherei) / Rezeption und Kritik der romantischen Naturforschung und Medizin an der Universität, auf Kongressen und in zeitgenössischen Publikationen.

Der Zeitraum der Romantik sollte grob mit den Jahreszahlen 1790 und 1830 begrenzt sein, eine weitergehende Vor- und Nachgeschichte, jedenfalls als allgemeines Thema des Symposions, außer Betracht bleiben.

Es war selbstverständlich, daß nur ein kleiner Teil dieser Sammlung von Motiven würde zur Sprache kommen können. Die Realität der Forschungssituation war allein die Basis einer Erwartung des Möglichen. Hier gab es viele Unbekannte im Konzept, die nicht im Voraus "kalkulierbar" waren. Über die Idee der Unternehmung und auch über die gewisse Zufälligkeit der Beiträge und ihrer Kombination ist in der "Begrüßung" noch einiges gesagt. Darauf sei verwiesen. Nichtsdestoweniger wurden im Gesamt der Vorlagen und in den Diskussionen die meisten Gegenstände des soeben zitierten Katalogs angesprochen.

Die Rahmenthemen sollten übrigens gerade nicht "Sektionen" bezeichnen mit einer Trennung der Wissenschaften und ihrer Gegenstände. Über die Vorstellung vom interdisziplinären Charakter des Symposions hieß es in der Ankündigung:

Ziel der Veranstaltung ist nicht eine Revue abgeschlossener und im wesentlichen bekannter Forschungsergebnisse. Vielmehr ist zu wünschen, daß neue Ansätze, noch nicht veröffentlichte Ergebnisse präsentiert und auf interdisziplinärer Ebene diskutiert werden. Insbesondere ist dabei gedacht an die Einbeziehung folgender Disziplinen: Literaturwissenschaft, Naturwissenschaften und Medizin, Geschichtswissenschaften (politische Geschichte, Rechtsgeschichte, Wirtschafts- und Sozialgeschichte), Philosophie, Theologie, Kunst- und Musikwissenschaft. Theoretische und methodische Reflexionen sowie allgemeine Fragen der Periodisierung und Epochengeschichtsschreibung sollten nur in der Anwendung am Beispiel vorgestellt werden. "Interdisziplinär" heißt nicht, daß Forschungsergebnisse verschiedener Fächer zum Thema Romantik nebeneinander gestellt und nur jeweils von den Fachleuten im engeren Sinne erörtert werden. Vielmehr geht es dem universalistischen Denken der Romantik selbst entsprechend um den Versuch einer Synthese disparater Forschungsansätze neuer Zeit. Die Beiträge der einzelnen Disziplinen sollten im Hinblick auf Berührungspunkte und wechselseitige Relevanz reflektiert werden und entsprechende Akzente setzen. Es ist also weniger an ganz spezielle Fachuntersuchungen gedacht, die eher auf Kongressen einzelner Wissenschaften oder zu einzelnen Problemen vorzutragen wären. Gleichwohl geht es um möglichst konkrete Hinwendung zu den Gegenständen der Fächer, bei der die verschiedenen Erkenntnisinteressen, Betrachtungsweisen und Forschungsschwerpunkte zur Geltung gebracht und konfrontiert werden.

Dieses Ziel ist, obzwar keineswegs perfekt, doch in einem über Erwarten effektiven und produktiven Maß erreicht worden. Dafür waren die Diskussionen von entscheidender Bedeutung. Der Erfahrene weiß, wie schwer sie - so paradox das klingen mag - auch dann wiederzugeben sind, wenn sie Wort für Wort, These für These, Replik für Replik reproduziert werden. In eigentümlicher Weise ist aus dem konservierten Wort der gesprochenen, aufgenommenen, erwiderten Rede das Leben entwichen; nicht nur die Gegenwart der Personen, selbst wenn man sie kennt oder sogar Augen- und Ohrenzeuge, ja Teilnehmer der Debatte war, ist dahin 
und papieren geworden, vielmehr hat selbst der intellektuelle Gehalt häufig Dimensionen eingebüßt, die in der Aura der dialektisch-dialogischen Situation und ihrer Träger durchaus gegenwärtig waren, und dies keineswegs nur als "irrationale" Zutat. Die Veranstalter haben sich deshalb in Übereinstimmung mit den Teilnehmern entschlossen, Zusammenfassungen der Diskussionen zu präsentieren, die lesbar sein sollen und die Einzelbeiträge integrieren ohne Nennung von Namen, mit Ausnahme natürlich der Referenten. Für die "Redaktoren," die für die Aufzeichnung der Diskussion und substantielle Arbeit bei der Herstellung des vorliegenden Bandes verantwortlich waren, kam es also darauf an, auf der Grundlage der vollständigen Tonbandauf nahmen jeweils so etwas wie einen selbständigen, zusammenhängenden "Essay" zu verfassen. Er sollte die Debatten nicht ergänzen, verändern oder erweitern, wohl aber komprimieren und ihre Tendenzen, Ergebnisse und Grenzen, gerade auch im Hinblick auf das Ziel des Interdisziplinären, erkennbar machen. Es ist dabei wohl kein Nachteil, daß die Berichte in Stil und Umfang leicht variieren. Auf Diskussionseinführungen der Referenten wurde dann Bezug genommen, wenn sie Ergänzungen der Vorlagen brachten. Nicht Opfer von Abbreviatur durften Material-Ergänzungen und -Nachträge in der Diskussion werden. Als eine solche Ergänzung ist auch die "Bibliographie der Sekundärliteratur zur romantischen Naturforschung und Medizin 1950-1975" von Dietrich v. Engelhardt zu betrachten. Da Literaturangaben aus diesem Bereich für Nicht-Naturwissenschaftler erfahrungsgemäß schwerer zugänglich sind, erschien es angebracht, die einschlägigen Titel in einigermaßen umfassender Weise zu dokumentieren.

Das Romantik-Symposion und seine Dokumentation, für deren Veröffentlichung im Hinblick auf die Vielzahl der Fächer ein Sonderband der Deutschen Vierteljahrsschrift für Literaturwissenschaft und Geistesgeschichte besonders geeignet erschien, sollen nicht als Abschluß, sondern als Anf ang, nicht als pompöse Lehre ex cathedra sondern als offenes Angebot, nicht als Paradigma sondern als Ermunterung zu kritischem Fortschreiten in der Romantik-Forschung verstanden werden.

Das Letzte, aber wahrlich nicht das Geringste in diesen "Vorbemerkungen" sei der wiederholte Dank an die Freunde und Kollegen, die als "Kurator" und "Redaktor" zu Vorbereitung, Organisation und Verlauf der Veranstaltung Entscheidendes beigetragen haben. Besonders genannt sei noch Herr Dr. Gerhart von Graevenitz (Tübingen), weil sein Name im folgenden nicht auftaucht. Er war eine tatkräftige und besonnene Hilfe und hat in allen Phasen der Planung und Durchfuihrung, nicht zuletzt auch bei der arbeitsreichen Redaktion dieses Bandes, unschätzbare Dienste geleistet.

Richard Brinkmann 